\title{
Les politiques de jumelage à la ville de Bouaké : quels discours pour le développement local?
}

\author{
Yéo Tenan ${ }^{a}$, Kouakou Kouaméb, Chantale Dali ${ }^{\mathrm{c}}$
}

RÉSUMÉ. Dans cet article, nous proposons de faire une analyse de l'impact du discours des politiques de jumelage sur le développement de la ville de Bouaké, plus particulièrement sur la reconstruction post-conflit de cette cité. Le discours des programmes de jumelage était à l'origine destiné à rapprocher les peuples dans une visée émulatrice de paix et d'barmonie. Toutefois, ce discours s'est étendu depuis quelques décennies à la sphère économique dans une vision d'action du discursif sur le social en promouvant les villes sur le marché désormais ouvert et mondialisé. Ainsi, à l'image de ses conscurs, le discours diplomatique de Bouaké prend une configuration particulière qui caractérise ses ambitions et ses attentes dans la coopération. Cependant, l'impact de ce discours sur le développement de la ville est faible et ne répond pas aux besoins prioritaires de la reconstruction post-conflit. Ce qui résulte de notre analyse est une inadéquation entre la vision vébiculée par le discours de la coopération et les actions qui découlent de celui-ci.

\begin{abstract}
In this article, we propose to examine the impact of the discourse of twinning policies on the development of the city of Bouake, more specifically on its post-conflict reconstruction. The discourse of twinning programs was originally intended to bring people together with the perspective of emulating peace and harmony. However, over the past few decades, this discourse has been extended to the economic sphere in a discursive vision of social action promoting cities now in a globalized and open market. Therefore, following the example of her twin sisters, the diplomatic speech of Bouaké takes a particular configuration that characterizes its ambitions and expectations in cooperation. However, the impact of this discourse on the development of the city is weak and does not meet the basic needs of post-conflict reconstruction. This creates a mismatch between the discourse and the actions that results from it.
\end{abstract}

\section{Introduction}

Cet article est inscrit dans la perspective d'une analyse de contenu des discours relatifs aux politiques de jumelage entre la ville de Bouaké (ville située au centre de la Côte d'Ivoire) et ses villes jumelles. Il cherche à dégager l'impact réel de ces discours sur le développement de cette cité.

Pour rappel, la propension des acteurs de la gouvernance locale à établir des relations internationales avec différentes cités a donné jour à ce qui est convenu d'appeler la « diplomatie des villes ». Cette diplomatie est d'un tout autre genre par rapport à celle de l'État. Les villes n'ayant pas un pouvoir régalien, cette diplomatie a vu son champ d'action s'élargir au cours des décennies pour couvrir des réalités très diverses. Caractérisant et balisant la portée de l'action internationale des cités, les acteurs de la gouvernance locale réunis à la conférence mondiale organisée sur la « diplomatie des villes » à La Haye du 11 au 13 juin 2008 , en donnent la définition suivante : «La diplomatie des villes est l'outil des gouvernements locaux et de leurs associations en vue de la promotion de la cohésion sociale, de la prévention des conflits, de la résolution des conflits et de la reconstruction postconflit, dans le but de créer un environnement stable dans lequel les citoyens peuvent vivre ensemble dans la paix, la démocratie et la prospérité »1. Ainsi, dans le cas spécifique de la ville de Bouaké, parler du discours de la «diplomatie des villes», revient, selon nous, à interroger l'impact de cette diplomatie dans un contexte

\footnotetext{
a Université Alassane Ouattara de Bouake

${ }^{\mathrm{b}}$ Université Alassane Ouattara de Bouake

c Professionnelle de recherche, Laboratoire de recherche et d'intervention sur le développement de l'entreprise dans les pays en développement, Université du Québec à Trois-Rivières
} 
post-conflit, caractéristique socio-économique actuelle de cette ville. En effet, si en situation ordinaire, l'objectif assigné et assimilé à la gouvernance décentralisée est le développement local, le contexte postconflit rend cette ambition plus accrue, plus urgente et plus large, en ce sens qu'il est davantage question de reconstruction que de construction. Dans un tel contexte, nous tenterons de montrer en quoi le discours de la diplomatie des villes est une construction socio-langagière dynamique et évolutive qui « sémiotise » des visions particulières. Nous jetterons par la suite un regard sur la configuration discursive et conceptuelle des différentes politiques de jumelage entreprises par la ville de Bouaké, selon que la ville jumelle soit du Sud ou du Nord. Pour finir, nous analyserons la portée sociétale de ce discours d'ouverture, en d'autres termes, sa performativité.

Notre étude s'appuie sur un corpus constitué de discours prononcés lors des cérémonies de signature et de renouvellement des protocoles d'accord de jumelage entre la ville de Bouaké et ses villes jumelles, consignés dans les archives de la ville. Sont aussi pris en compte les articles de la presse nationale et internationale qui font le relais médiatique des actions de la ville de Bouaké à l'international et au national.

\section{Le discours de la diplomatie des villes : un artefact dynamique et évolutif}

Le discours sur les politiques de jumelage des collectivités territoriales a vu le jour dans le sillage de la mise en place des stratégies des institutions qui avaient pour but de garantir la paix entre les peuples après le traumatisme causé par la seconde guerre mondiale (Zelinsky, 1991; Petiteville, 1996; Richier, 2014). Les jumelages étaient à cette époque uniquement le résultat d'initiatives spontanées d'élus locaux qui avaient particulièrement à cœur de rapprocher les peuples dans un contexte historique précis, et ce, dans les débuts de la construction européenne (Richard, 2012). Partant de l'a priori que l'ignorance et la méconnaissance de l'autre sont les terreaux fertiles à l'émergence des guerres, le discours de la diplomatie des villes prônait de prime abord l'ouverture sur le monde, la solidarité et les valeurs humanistes et universelles dans l'aspiration des peuples à se connaître et à se comprendre. Cette construction post-conflit s'était assigné la mission de rapprocher les hommes en créant un espace de rencontre interculturelle.

Dans cet élan humaniste, plusieurs mouvements de coopérations décentralisées ont émergé en Europe de l'Ouest, notamment, conduits par un idéal de fraternité à la base qui voulait dépasser les oppositions historiques liées aux politiques étatiques. Au nombre de ces mouvements, nous pouvons mentionner le Conseil des communes en Europe (CCE), fondé en 1951 (devenu le Conseil des communes et des régions d'Europe en 1984) et la Fédération mondiale des Villes jumelées (FMVJ) en 1957 (devenue en 1988, Fédération mondiale des Citées unies et des Villes jumelées). Ce dernier mouvement, fondé par d'anciens résistants francobritanniques, s'était donné pour objectif de faire apprendre à chaque enfant une autre langue que la sienne afin d'élargir la compréhension mutuelle. Ainsi, les jeunes français devaient apprendre la langue anglaise et les jeunes anglais, la langue française.

Dans la même veine, des actions sont menées par les élus locaux des collectivités territoriales européennes, en l'occurrence françaises et allemandes pour la réconciliation franco-allemande. Dans ce contexte de la réconciliation et de la reconstruction européenne, maires et citoyens étaient déterminés à œuvrer pour que plus jamais le monde ne soit plus déchiré par la guerre. Il apparaît alors que le seul moyen de progresser sur le plan des relations internationales et d'apaiser les rancœurs est de tisser des liens parmi les communes, d'établir des relations de fraternité entre les citoyens par l'échange de connaissances, d'expérience ou de savoir-faire dans les domaines de la vie locale. Ainsi, la réconciliation se voit désormais construire par « le bas », c'est-à-dire par les peuples directement et par leurs structures représentatives grâce aux partenariats entre les communes et les villes, autrement les jumelages.

Toutefois, en partant de la définition que les acteurs de la diplomatie des villes donnent à cette notion, nous remarquons une évolution dynamique et multisectorielle de l'action internationale des villes. Les objectifs de la diplomatie des villes limités aux questions de sécurités internationales, à la prévention des conflits, à la gestion des conflits et au rétablissement de la paix, s'ajustent dorénavant avec de nouveaux objectifs plus mouvants dont la 
《 reconstruction post-conflit » et la "prospérité », des questions éminemment économiques.

Le discours de la diplomatie des villes devient ainsi porteur d'une coopération de proximité pour le développement. Il se mût dorénavant en canal d'actions concrètes qui répondent aux besoins des populations. En effet, les gouvernants locaux s'occupant de la coopération internationale se sont intéressés à l'action économique dès les années 1980, c'est-à-dire à partir du moment où les communes ont commencé à intervenir massivement en faveur des entreprises. Au premier chef de la stratégie économique du territoire se trouvait la volonté d'attirer des investissements étrangers. On voit alors apparaitre la mise en place des premières formes d'aides à l'implantation d'entreprises comme l'instauration de primes ou encore de soutiens techniques (Richard, 2012) ${ }^{2}$. Fort de cette dynamique, le cadre de la diplomatie des villes allait enfin se transformer en un véritable lieu de signature de contrats commerciaux entre entreprises de communes jumelées lors des foires et salons spécialisés. Par ailleurs, l'introduction d'une dimension économique dans les jumelages a contribué à revaloriser l'image des fonctionnaires en charge de l'international, domaine qui jouissait auparavant d'une image uniquement folklorique. La conclusion des contrats commerciaux a aussi permis de légitimer l'action et même l'existence de ces services qui pouvaient désormais mettre en avant leur contribution au développement du territoire (Vion, 2001).

Avec la mondialisation et l'ouverture des marchés jalonnés de crises successives, les villes et les cités font aussi leur entrée dans la compétition internationale, faisant de cette course effrénée un crédo de plus en plus indispensable pour leur développement économique et social. Ainsi, dans leur quête pour le développement, les villes vont multiplier les approches de coopération, ce qui leur donne une plus grande visibilité internationale. Ce nouveau type de discours à visée exclusivement économique instaure la construction de réseau dynamique faisant la part belle à l'attractivité des métropoles. Les changements qui interviennent à l'échelle mondiale sont souvent ressentis localement, qu'il s'agisse de la mondialisation de l'économie, des changements climatiques ou de la situation démographique. Par définition, la diplomatie des villes est par excellence un espace de partage de connaissances, des expériences et des bonnes pratiques. Il est donc évident que dans les échanges entre les villes du Nord et les villes du Sud, la diplomatie permet souvent aux collectivités locales d'exploiter de multiples possibilités à travers des projets novateurs qui concourent à la création d'emplois et à l'établissement durable d'entreprises. Ces actions diverses peuvent prendre la forme d'échange très ciblés dans différents domaines comme la gestion urbaine, l'environnement, le développement durable ou encore le transfert de technologie. Dans ce contexte, la diplomatie des villes tout en élargissant son champ d'action génère un discours dynamique qui appelle la valorisation de certains concepts qui vont déterminer sa configuration dans une vision développementaliste où transparaissent des divergences de type Nord/Sud ou Sud/Sud.

\section{Configuration discursive des politiques de jumelages de la ville de Bouaké : divergence Nord/Sud et Sud/Sud}

Les collectivités territoriales formulent des discours d'ouverture qui laissent transparaitre les stratégies qu'ils mettent en place pour atteindre les objectifs de leurs politiques de coopération. La ville de Bouaké, en sa qualité de deuxième métropole de la Côte d'Ivoire après Abidjan, a très tôt adopté une politique d'ouverture dans la droite ligne de la configuration sociale qu'elle s'était assignée, c'est-àdire une ville à la croissance économique forte en relation avec sa situation géographique de ville carrefour. C'est dans cette perspective que, bien qu'étant à l'époque ville d'une colonie française, elle signa son premier accord de jumelage en 1958 avec la ville française de Villeneuve-sur-Lot. Ce premier accord de jumelage va ouvrir la voix à plusieurs autres accords avec des villes reparties dans le monde entre l'hémisphère nord et l'hémisphère sud. Cette ouverture de la ville de Bouaké sur le monde s'accompagne d'un discours dynamique qui se construit en relation avec les intentions et les intérêts réciproques des partenaires de l'échange. À la suite de Charaudeau (2004) et Bourdieu (1982), nous dirons que la forme que prend ce discours met en exergue sa propre sémiotisation mais aussi une idéologie intrinsèquement définie et conduite par des intérêts économiques. En effet, l'action socio-langagière que la ville de Bouaké déploie, justifie et renforce les motifs des résultats qu'elle espère de ses relations dans la diplomatie des villes en même temps qu'elle définit sa position par rapport aux autres villes. La liste de la dizaine de villes 
et communes jumelées à Bouaké a une répartition géographique quasiment équitable avec autant de villes jumelées au Nord qu'au Sud. Cependant, on peut dire que les différents concepts qui structurent le discours de la ville de Bouaké donnent un aperçu de la nature des échanges qui s'établissent entre elle et ses partenaires. Ce réseau conceptuel codifie les buts actionnels que s'assigne cette cité dans une finalité bénéfique de ses politiques de jumelage.

Ainsi, dans le cadre des relations de jumelage développées par la ville de Bouaké avec d'autres villes de l'hémisphère sud, en l'occurrence, des villes de pays africains, la majorité des accords signés ne prennent en compte que le domaine culturel. Alors que dans le même cadre des politiques de jumelage, les relations qu'elle se permet d'établir avec les villes de l'hémisphère nord, notamment, des villes européennes vont au-delà du culturel. Ces relations tiennent surtout compte de l'économie, de la santé et de l'éducation. Ces domaines éminemment stratégiques pour le développement local, l'économie, la santé et l'éducation semblent être au cœur des préoccupations des autorités municipales de Bouaké, surtout dans la politique de reconstruction post-conflit. Le tableau ci-dessous nous donne une vue d'ensemble de la configuration du discours des politiques de jumelages de la ville de Bouaké, en lien avec les différents intérêts recherchés.

\begin{tabular}{|c|c|c|}
\hline $\begin{array}{c}\text { Commune ou villes } \\
\text { partenaires }\end{array}$ & Hémisphères & Domaines d'échanges \\
\hline Villeneuve-sur-Lot/ France & Nord & $\begin{array}{l}\text { Culturel, économique, } \\
\text { santé }\end{array}$ \\
\hline Reutlingen/ Allemagne & Nord & $\begin{array}{l}\text { Éducatif, économique, } \\
\text { culturel, santé }\end{array}$ \\
\hline $\begin{array}{c}\text { Bobo-Dioulasso/ Burkina- } \\
\text { Faso }\end{array}$ & Sud & Culturel \\
\hline Ziguinchor/Sénégal & Sud & Culturel \\
\hline Brescia/Italie & Nord & $\begin{array}{l}\text { Éducatif, culturel, } \\
\text { économique, santé }\end{array}$ \\
\hline Beer-Shev'a/ Israël & Nord & $\begin{array}{l}\text { Éducatif, culturel, } \\
\text { économique, santé }\end{array}$ \\
\hline Agadir/Maroc & Sud & $\begin{array}{l}\text { Éducatif, culturel, } \\
\text { économique, santé }\end{array}$ \\
\hline Mopti/Mali & Sud & Culturel \\
\hline Porto/Portugal & Nord & $\begin{array}{l}\text { Éducatif, culturel, } \\
\text { économique, santé }\end{array}$ \\
\hline Bodokro/Côte d'Ivoire & Sud & Culturel \\
\hline Odienné/ Côte d'Ivoire & Sud & Culturel \\
\hline
\end{tabular}

Tableau 1 - Tableau des stratégies de jumelage de la ville de Bouake

Source : Les auteurs 
Ce réseau conceptuel illustre bien dans ce tableau la configuration particulière que prend le discours de la ville de Bouaké en matière de diplomatie des villes; toute chose qui détermine son positionnement entant que sujet communiquant dans cette organisation spécifique du monde. Dans ce sens, les domaines que couvrent les relations de la coopération décentralisée entreprise par la ville de Bouaké sont proportionnels à la puissance économique attribuable aux pays d'origine de la ville jumelle : plus le dynamisme du pays est avéré, plus vaste sont les domaines inclus dans la coopération alors que la réciproque n'est pas toujours vraie.

Le discours de la diplomatie des villes prend forme dans ce contexte dans un inter-discours qui en définit les fondements et la base idéologique. De prime abord, il est admis au regard de cet interdiscours qu'il existe une fracture économique entre les pays du Nord, industrialisés et développés et les pays du Sud, pays sous-développés ou en voie de développement, ou pauvres. Dans la logique du partage et de l'échange, le tiers, ayant moins de ressources, s'attend naturellement à recevoir plus du tiers plus nanti. Cette logique, si elle n'est pas formellement inscrite dans la définition de la diplomatie des villes, elle n'en structure pas moins la manifestation de ces politiques et le discours qui les accompagnent, comme le montre le tableau cidessus.

Pour étayer cette hypothèse, nous allons nous appuyer sur l'exemple de la ville d'Agadir au Maroc. Bien qu'étant un pays du Sud, la nature des relations qu'entretient la ville de Bouaké avec cette dernière diffère substantiellement de celle qu'elle a avec les autres villes du Sud. Pour cause, le Maroc a un dynamisme économique qui lui donne une certaine avance sur la Côte d'Ivoire. Dans les rapports économiques sur le continent africain, le Maroc occupe la $5^{e}$ place des économies les plus performantes; ce pays serait la 3 e puissance économique du continent selon une étude du Think Tank Londonien (Legatum Institute, 2016) tandis que la Côte d'Ivoire est classée à la 20 e place selon le même rapport. De plus, le Maroc se positionne comme l'un des partenaires économiques les plus dynamiques de la Côte d'Ivoire avec des investissements dans plusieurs domaines stratégiques de son économie (CEPICI, 2016) ${ }^{3}$. En rapport avec ce qui précède, le discours de la ville de Bouaké, dans ce cas particulier, prend la configuration du discours qu'il énonce dans ses relations avec les villes des pays du Nord.

En résumé, la configuration discursive de la ville de Bouaké dans sa politique de coopération augure d'une sémiotisation propre qui donne aux sujets communicants un positionnement dans la relation décrite. Cependant, ce discours de la coopération internationale de la ville de Bouaké est-elle en adéquation avec la vision qui lui est assignée, c'est-àdire une amélioration qualitative dans la vie de cette cité? En d'autres propos, ce discours a-t-il un impact réel sur le développement de cette cité?

\section{Entre vision et action : quel impact des politiques de jumelage sur le développement local de Bouaké?}

Si le discours de la diplomatie des villes a connu un mouvement dynamique pour se munir de prérogatives pragmatiques et économiquement productives, il est de ce fait intéressant d'interroger l'impact réel de ce discours sur la réalité particulièrement dans le cas de la ville de Bouaké. En effet, les acteurs de la diplomatie des villes sont peu à peu allés au-delà du cadre traditionnel et symbolique des jumelages pour les reconvertir en un instrument qui leur permettrait d'obtenir une plus-value dans le domaine de la compétitivité des villes et de l'attractivité du territoire.

Plusieurs travaux de recherche ont mis en exergue l'apport de l'action extérieur des collectivités territoriales dans le développement local. Au nombre de ceux-ci, nous avons les travaux d'orientation économique telle que l'approche de Ogandaya (2014), qui juge de la réciprocité en termes de développement des politiques de coopération décentralisée entre les collectivités territoriales françaises et africaines; ou encore celle à visée sociologique de Savitch et Kantor (2002), qui se penche sur l'élaboration des stratégies d'internationalisation des villes. C'est dans ce vaste champ de recherche sur l'action diplomatique des collectivités territoriales que s'inscrit la posture «objectivante » de ces relations qui mettent la focale sur les infrastructures (Chombard-Gaudin, 1995; Chombard-Gaudin et Ribault, 1996; Kukawka, 1994). Cette approche a été introduite par le centre de recherche scientifique GIP-Reclus qui a établi un certain nombre de critères devant permettre de juger le degré d'insertion à l'internationale de la 
ville. Ce degré d'internationalisation relève de l'impact de ces relations sur le développement local de façon transversale. Saldatos (1991) décrit le cadre de ces relations comme :

Un lieu privilégié pour mener à bonne fin des opérations intellectuelles, commerciales, technologiques, culturelles et politiques entre partenaires de nations différentes à condition que ces opérations soient à ce point, répétitives et nombreuses, qu'elles créent des échanges permanents d'hommes, d'idées, de produits, de recherche, devenant constitutifs d'une mission, d'une animation et presque d'une raison d'être (Saldatos, 1991, p. 158).

Dans cette perspective, l'action extérieure des villes se positionne comme une importante partie de l'activité de celles-ci dans leur recherche de ressources pour le développement. La ville de Bouaké en étant dans le peloton de tête des villes de la Côte d'Ivoire et ayant perçu l'importance des relations internationales avec d'autres collectivités territoriales à travers le monde envisageait cette ouverture aux autres comme un canal pour sa promotion et son développement. Bien que prospère dans la foulée et l'élan du miracle économique ivoirien ${ }^{4}$, la ville a connu une longue période de crise économique aggravée par les différentes crises militaro-politiques depuis les années 2000 dont le paroxysme a été la guerre civile de $2002^{5}$. L'économie déjà fragilisée de la ville sera ainsi complètement endommagée, laissant la ville dans un état déplorable et presqu'en ruine. Avec la fermeture des unités de production les unes après les autres, l'industrie qui fut longtemps un secteur florissant et dynamique de la ville surtout celle de l'agroalimentaire et du textile n'est plus que l'ombre d'elle-même. Malgré le rétablissement définitif de la paix en 2011, cette industrie peine à se relever. Sur la trentaine d'usines que comptait la zone industrielle de la ville, seules cinq continuent de fonctionner. La grande unité industrielle du textile qui jadis comptait 5000 employés ne compte aujourd'hui que 200 qui travaillent par intermittence (Yasseu, 2016).

Dans un tel contexte, le discours économiste de l'action extérieure de Bouaké surtout à l'endroit de ses partenaires de l'hémisphère nord ne semble pas avoir de répercussion réelle sur l'amélioration de l'économie et des conditions de vie de la cité. L'attractivité procurée par les politiques de jumelage reste attendue dans la renaissance de la ville.
Certes, des échanges existent entre Bouaké et ses partenaires, à l'image de ceux établis avec Villeneuve-sur-Lot en France et Reutlingen en Allemagne, qui sont les partenaires avec lesquels Bouaké a une coopération plus active et dynamique. Les échanges dans le cadre des politiques de jumelage de Bouaké avec ses deux villes sœurs jumelles serviront à illustrer nos propos dans cette section. Si l'existence de ces échanges ne fait pas de doute, ceux-ci sont cependant loin de répondre aux besoins de la renaissance post-conflit de la ville. En effet, Villeneuve-sur-Lot, selon Michel Barjou, membre du comité de jumelage VilleneuveBouaké, «a pris l'habitude d'affréter tous les deux ans vers Bouaké un ou plusieurs conteneurs de médicaments, de matériel hospitalier obsolètes, de machine outils et de livres» (Lebrese, 2000). De même, une délégation des autorités municipales de Villeneuve, en séjour à Bouaké en mars 2018, a visité des centres de santé de la ville pour faire un «audit lié aux problèmes de santé des populations les plus défavorisées » dans l'objectif «d'apporter un maximum de mieux être à ces populations » (Abidjan.net 2018). Quant à la coopération avec la ville de Reutlingen, elle s'étend sur plusieurs domaines, notamment la «formation continue des professeurs d'Allemand », le "partenariat scolaire » et le domaine culturel avec des échanges sur le plan du théâtre. Le point culminant de cette coopération entre Bouaké et Reutlingen, sur le plan économique et social, est le projet de réalisation de biodigesteurs, producteurs d'énergie propre «utilisable dans le cadre de la cuisine des cantines scolaires de neuf (09) établissements primaires d'un coût total de 162 millions de FCFA » (Schreiner, $2015)^{6}$.

Ces deux exemples illustrent la dynamique des échanges entre Bouaké et ses villes jumelles. Toutefois, nous pouvons avancer que ces échanges concernent plus à l'assistance humanitaire; qu'ils ne prennent pas en compte les besoins économiques. En effet, la reconstruction post-conflit a ouvert plusieurs axes prioritaires pour la relance de la ville. C'est ainsi que selon son site Internet, la mairie ${ }^{7}$ envisage de reconstruire le marché centrale, ravagé par un incendie, il y a plusieurs décennies. À cela s'ajoute la réhabilitation des ouvrages de drainages et de gestion des ordures ménagères, la construction d'école et surtout la relance de l'activité industrielle et commerciale. Malgré cette évidence notoire, les chantiers prioritaires de la relance de la 
ville ne sont nullement pris en compte dans les actions découlant de la coopération bilatérale entre la ville et ses partenaires. Dès lors, on peut conclure que ce constat laisse entrevoir une nette divergence entre la vision véhiculée dans le discours de la coopération et les actions qui découlent de celui-ci. Il s'instaure ainsi des rapports de non-effectivité entre les activités sociales et les unités sémiotiques qui les médiatisent.

Cette non-effectivité des rapports discours-actions s'expliquent par diverses réalités liées à l'histoire contemporaine de la ville de Bouaké. D’une part, les différents remous sociaux créés par les crises successives dont la ville a été victime ont considérablement ralenti et compromis les rapports avec l'extérieur, en particulier dans le cadre du jumelage. D'autre part, la ville a connu une explosion démographique qui a fait passer sa population de quelques milliers d'habitants à l'orée de l'indépendance du pays en 1960 à 1500000 habitants aujourd'hui (RGPH, 2014). Cette réalité démographique engendre de nouveaux types de défis économiques à relever particulièrement sur le plan de la création d'emplois. La combinaison de ces deux réalités met la ville au-devant de défis colossaux qui ne trouvent concrètement pas de voie de solution dans la mise en œuvre du discours des politiques de jumelage. Aussi, la modestie des villes partenaires avec lesquelles Bouaké a une coopération active augure des insuffisances et de l'inadéquation de ce discours aux réalités sociales. Dans ce sens, il y a un véritable contraste entre la ville de Bouaké et sa première sœur jumelle, Villeneuvesur-Lot. Tandis que la première compte 1500000 habitants, la seconde n'en compte que 22 686, d'après les chiffres de l'année 2015. La même remarque est faite pour la ville de Reutlingen qui comptait 114300 habitants selon les chiffres de la mairie en 2015. La ville de Bouaké a amorcé la voie de la métropolisation pendant que ses partenaires les plus dynamiques de l'Occident que sont Villeneuve-sur-Lot et Reutlingen, sont encore à l'étape de petite et moyenne ville. Toute chose qui crée un déséquilibre des niveaux de besoins et partant, de la nature des échanges dans le cadre de la diplomatie des villes. Il se crée dans une telle configuration un réel fossé entre le «dire» et le "faire »; une inadéquation entre le discours et l'action sociale. L'impact du discours des politiques de jumelage sur le développement économique et social de la ville de Bouaké reste largement en dessous de ses besoins réels, mais aussi des potentialités qu'elle peut offrir à ses consœurs. Au nombre de ces potentialités, on pourrait citer par exemple le cas de la médecine traditionnelle, une compétence que la ville peut partager avec les villes jumelles et leur en faire bénéficier les vertus. Une politique d'échanges de connaissances qui permettrait à terme de moderniser ce secteur.

\section{Conclusion}

Il s'est agi pour nous dans ce parcours analytique de mettre en lumière les rapports discours-actions dans le contexte social des politiques de jumelage entreprises par la ville de Bouaké.

Ainsi, il est paru en premier lieu que le discours de la diplomatie des villes est une entité dynamique et mouvante qui évolue dans le temps en s'octroyant de nouvelles prérogatives qui contribuent largement au rayonnement social et à l'attractivité économique des villes. Ce discours est parti des ambitions humanistes et interculturelles de rencontre des peuples pour devenir un instrument de l'expression des visions et des visées économistes et développementalistes.

Nous avons vu en second lieu que le discours de la diplomatie des villes adopté par Bouaké est le réceptacle des visions et des attentes de cette dernière dans la coopération bilatérale. Dans ce cadre, le discours de la ville de Bouaké prend une configuration en fonction de la ville partenaire en présence, selon que celle-ci soit du Nord ou du Sud. Ce discours diplomatique s'enrichit d'un interdiscours évident et détermine la position des partenaires, en l'occurrence, leur puissance d'action dans la coopération bilatérale.

Enfin, l'analyse a mis en exergue les limites dans la sphère sociale de la ville de Bouaké de la portée du discours des politiques de jumelage. En effet, dans la situation particulière de la reconstruction postconflit de la ville de Bouaké, les axes prioritaires font référence aux besoins d'ordre économique. La satisfaction de ces besoins passe par une implication directe de l'action tant intérieure qu'extérieure dans des domaines tels que l'industrie, le commerce, les infrastructures, etc. Toutefois, les actions découlant de la politique extérieure de la ville ne couvrent nullement ces secteurs indispensables à la relance de la cité de Bouaké. Ces limites mettent à mal les rapports entre le «dire» et le 
«faire» et démontrent du minime impact des apports du jumelage dans la reconstruction postconflit de la ville de Bouaké autrefois cité prospère et dynamique.

Pour finir, nous dirons que si la ville de Bouaké a très tôt compris la grande occasion que représente les politiques de jumelage pour sa promotion et son développement, la nature et les évolutions de ces besoins sociaux lui imposent de s'ouvrir à d'autres partenaires proportionnellement à ses visions et ses attentes. Ainsi, en se repositionnant comme une cité cosmopolite aux valeurs multiples, Bouaké gagnerait à redéfinir son action sur le plan de la diplomatie des villes en coopérant avec d'autres villes économiquement plus dynamiques; d'autres villes prêtes à partager dans cette politique du donner et du recevoir les potentialités et les valeurs dont Bouaké dispose afin qu'elle cesse de paraître comme une partenaire qui ne fait que profiter des autres.

\section{NOTES}

1 Première conférence mondiale organisée sur le thème de la « diplomatie des villes » à La Haye du 11 au 13 juin 2008. Agenda de La Haye sur la diplomatie des villes. Repéré à www.cities-localgoverments.org.

2 Cette politique a notamment été menée en France sous la houlette de la Délégation à l'Aménagement du Territoire et à l'Attractivité régionale (DATAR).

3 Selon le Centre de Promotion des Investissements en Côte d'Ivoire (CEPICI, 2016) en 2015, les opérateurs économiques marocains représentaient $22 \%$ des entreprises agréées par le CEPICI se classant ainsi à la deuxième place des investissements étrangers dans le pays après les entreprises françaises.

4 Le miracle économique ivoirien est le qualificatif donné à une période de prospérité qu'a connue la Côte d'Ivoire dans les années 1960-1970.

5 Cette guerre a éclaté après l'échec d'une tentative de coup d'État le 19 septembre 2002. La ville de Bouaké servira alors de base aux troupes rebelles pendant huit ans.

6 Environ 304560 dollars américains.

7 Projet d'aménagement de la zone marchande du centre ville de Bouaké. Repéré à www.mairiedebouake.ci

\section{RÉFÉRENCES}

Abidjan.net (2018). Les villes de Bouaké et de Villeneuve-sur-Lot célèbrent 60 ans de jumelage. Repéré à https://news. abidjan.net/h/632179/html

Bourdieu, P. (1982). Ce que parler veut dire. Paris, France: Fayard.

Charaudeau, P. (2004). Comment le langage se noue à l'action dans un modèle socio-communicationnel du discours. De l'action au pouvoir. Dans L. Filliettaz (Éd.) Les modèles du discours face au concept d'action: actes du 9ème Colloque de Pragmatique de Genève et Colloque Charles Bally. (pp. 151-175). Genève, Suisse: Université de Genève.

CEPICI (2016). Un bilan 2015 positif pour le CEPICI. Repéré à http://www.cepici.gouv.ci/web/docs/newsletter---fevrier2016.pdf

Chombard-Gaudin, C. (1992). Pour une histoire des villes et communes jumelées. Vingtième Siècle, revue d’histoire, 35, 60-66. doi :10.3406/xxs.1992.2565

Chombard-Gaudin, C. (1995). Les dates et des lieux : les jumelages en bourgogne, Languedoc-Roussillon et Limousin. Mappe Monde, 4, 11-16. Repéré à https:/ /www.mgm.fr/PUB/Mappemonde/M495/JUMELAGE.pdf

Chombard-Gaudin, C. et Ribault, S. (1996). Les jumelages du Calvados. Repéré à file:///C:/Users/utilisateur/Downloads/Les_jumelages_du_Calvados.pdf

Kukawka, P. (1994). Politiques de communication et d'image des villes de l'arc Atlantique. Communication et Organisation, 6, 44-60. Repéré à https://journals.openedition.org/communicationorganisation/1737?lang=en

Lebrese, R. (2000, 20juillet). 43 ans de jumelage. Repéré à https://www.ladepeche.fr/article/2000/07/20/77595-43-ans-dejumelage.html

Legatum Institute (2016). The Africa Prosperity Report 2016. Repéré à http://media.prosperity.com/2016/pdf/2016-

AfricaReport-web.pdf 
Ogandaya, N. C. (2014). La coopération décentralisée entre collectives territoriales françaises et africaines : les leviers de limplication et de la réciprocité (Thèse de doctorat inédite). Université de Lille 1, France. Repéré à https:// ori-nuxeo.univlille1.fr/nuxeo/site/esupversions/3cc131ed-0abe-457a-8078-078780d63860

Petiteville, F. (1996). La coopération décentralisée : les collectivités locales dans la coopération Nord-Sud. Paris, France: L'Harmattan.

Richard, S. (2012). La rationalisation des coopérations bilatérales au sein de l'action extérieur de la métropole lyonnaise : enjeux, manifestations et limites (Mémoire de maîtrise inédit). Université de Lyon. Repéré à http://doc.sciencespolyon.fr/Ressources/ Documents/Etudiants/Memoires/Cyberdocs/MFE2012/richard_s/pdf/richard_s.pdf

Richier, W. (2014). Les jumelages entre Guerre froide et enjeux locaux. Dans P. Buton, O. Büttner et M. Hastings (Éds.), La Guerre froide vue d'en bas. (pp. 131-149). Paris, France: CNRS Éditions. Repéré à https:// halshs.archives-ouvertes.fr/ halshs-01260097/document

RGPH (2014). Résultats Globaux, recensement général de la population et de l’habitat 2014. Repéré à http://www.ins.ci/n/Resultats\%20GBOBAUX2014.

Saldatos, S. (1991). Les nouvelles villes internationales : profil et planification stratégique. Aix-en-Provence, France: Serdeco.

Savitch, H. V. et Kantor, P. (2002). Cities in international market place: the political economy of urban development in North America and Western Europe. Princeton, NJ: Princeton University Press.

Schreiner, T. (2015). Une amitié sur deux continents. Repéré à https://www.goethe.de/ins/ci/fr/kul/mag/20749354.html

Vion, A. (2001). Au-delà de la territorialité, l'internationalisation des villes ? Note sur quelques déplacements de frontière de la politique. Annales Françaises de Relations Internationales, 2, 363-375. Repéré à http://www.afri-ct.org/wp-content/uploads/2006/03/vion2001.pdf

Yasseu, R. (26 septembre, 2016). Bouaké SOS pour le tissu industriel.Politik.Afrique.info. Repéré à http:// politikafrique.info/bouake-sos-tissu-industriel/

Zelinsky, W. (1991). The twinning of the world: sister cities in geographic and historical perspective. Annals of the Association of American Geographic, 81(1), 1-31. Repéré à http://www.jstor.org/stable/2563668 